\title{
Impact of the COVID-19 pandemic on emergency CT head utilization in Ontario-an observational study of tertiary academic hospitals
}

\author{
Minu Agarwal ${ }^{1,2} \cdot$ Amar Udare $^{1,2} \cdot$ Abdullah Alabousi $^{3} \cdot$ Christian B. van der Pol $^{1,2} \cdot$ Lucas Ramonas $^{4} \cdot$ Ken Mascola $^{1} \cdot$ \\ Britney Edmonds ${ }^{5} \cdot$ Milita Ramonas $^{1,2,6}$ (D)
}

Received: 23 August 2020 / Accepted: 24 September 2020 / Published online: 6 October 2020

(C) American Society of Emergency Radiology 2020

\begin{abstract}
Purpose To determine the impact of the Coronavirus disease-2019 (COVID-19) pandemic on the volume, indications, and results of CT heads performed in the emergency department (ED) at Canadian tertiary academic centers in Ontario.

Methods A retrospective review of consecutive CT head examinations ordered through the ED during the early stages of the COVID-19 pandemic in Canada (March 12-April 8, 2020) was performed and compared with that during the pre-COVID-19 period (February 12-March 10, 2020). CT reports were reviewed for the exam indication and the presence of predefined acute findings. A two-sample $t$ test was utilized to compare the cohorts. Daily averages were calculated for all measures to control for the sample size difference between each period. A $p$ value $<0.05$ was considered significant.

Results Of the total 751 CT head reports, 290 (38.6\%) were ordered during the pandemic and 461 (61.4\%) were ordered prepandemic. The average daily volume of CT head orders decreased significantly during COVID-19 compared with that during pre-COVID-19 (10.4 scans/day vs 16.5 scans/day; $p=0.001)$. In terms of indications, the frequency of "non-traumatic ICH" was significantly lower during COVID-19 compared with that during pre-COVID-19 $(p=0.01)$. Also, there was a significant increase in acute findings on CT during COVID-19 compared with that during pre-COVID-19 $(p=0.001)$.

Conclusion The decreased volume of emergency CT heads performed during the COVID-19 pandemic was associated with a significant increase in the proportion of cases with acute findings. This could be a result of the pandemic's influence on the health-seeking behavior of patients as well as the decision-making process of ordering clinicians.
\end{abstract}

Keywords Tomography $\cdot$ X-ray computed $\cdot$ Head $\cdot$ Emergency service $\cdot$ Hospital $\cdot$ Coronavirus $\cdot$ Pandemics $\cdot$ Diagnostic tests $\cdot$ Routine $\cdot$ CT head

\author{
Milita Ramonas \\ ramonas@hhsc.ca \\ Minu Agarwal \\ agarwalmi@hhsc.ca \\ Amar Udare \\ udare@hhsc.ca \\ Abdullah Alabousi \\ aalabous@stjoes.ca \\ Christian B. van der Pol \\ vanderpolc@hhsc.ca \\ Lucas Ramonas \\ lramo009@uottawa.ca \\ Ken Mascola \\ mascola@hhsc.ca
}

Britney Edmonds

martinbrit@hhsc.ca

1 Department of Radiology, Hamilton Health Sciences, 711 Concession Street, Hamilton, ON L8V 1C3, Canada

2 Department of Radiology, McMaster University, Hamilton, Canada

3 St. Joseph's Healthcare Hamilton, Hamilton, Canada

4 Undergraduate Studies, Health Sciences, University of Ottawa, Ottawa, Canada

5 Hamilton Niagara Haldimand Brant Regional Cancer Program, Hamilton, Canada

6 Department of Diagnostic Imaging, Juravinski Hospital and Cancer Centre, Hamilton Health Sciences, 711 Concession Street, Hamilton, Ontario L8V 1C3, Canada 


\section{Introduction}

The Coronavirus (COVID-19) outbreak that began in Wuhan, China, was labeled a pandemic by the World Health Organization (WHO) on March 11, 2020 [1]. This coincided with the city of Hamilton, Ontario, witnessing its first confirmed case, when a healthcare worker tested positive for the virus [2]. Within a week as the number of cases across the province grew, Ontario hospitals were directed to ramp down nonemergency ambulatory, surgical, and diagnostic programs to support the containment of COVID-19 cases in Ontario and to preserve hospital beds and resources for patients who contracted the virus [3].

Most radiologists at our department perceived an associated decrease in the computed tomography (CT) studies of the head ordered through the emergency department (ED) since the first case was identified in Hamilton. A substantial decrease in the daily ED visits since the pandemic has been confirmed at other institutions by multiple recent studies all over the globe [4-11]. Bres Bullrich et al. found a $20 \%$ decrease in the number of code strokes in the beginning of the pandemic compared with that in a similar period 1 year prior [11]. While using neuroimaging as a proxy to emergency services provided, Kansagra et al. revealed a 39\% decrease in the use of stroke imaging in the USA during the early pandemic period compared with that during the pre-pandemic period [12]. Such a decline in urgent healthcare access raises concerns for decreased or delayed presentations for life-threatening conditions like stroke. Moreover, the overall decline in daily ED visits may be related to patients with chronic and/or more minor complaints choosing to delay their presentation. It may be that the fear of contracting a new disease surpasses the concern for an existing illness.

Knowledge of the indications that are less likely to present to the ED or less likely to be imaged during such exceptional circumstances can be utilized by policy-makers in more appropriate planning of the healthcare directives and public messages related to healthcare utilization going forwards. Using neuroimaging as a surrogate, we aimed to evaluate the impact of the pandemic on emergency health-seeking behavior at our institution and to identify the indications that were responsible for the expected decline. The purpose of our study was to determine the impact of the Coronavirus disease-2019 (COVID-19) pandemic on the volume, indications, and results of CT heads ordered through the ED at our institution in comparable time periods before and after the first case of COVID-19 was diagnosed in Hamilton, Ontario.

\section{Methods}

\section{Study design}

A retrospective observational study was performed. Consecutive CT head examinations ordered through the ED at two tertiary care hospitals, Juravinski Hospital and Cancer Center (JHCC) and Hamilton General Hospital (HGH) were identified on the Picture Archiving and Communications System (PACS) workstation (Centricity PACS 6.0, GE Healthcare, Chicago, IL, USA) during a 4-week period of the early COVID-19 pandemic (March 12, 2020-April 8, 2020) and compared with the same duration in the preCOVID-19 period (February 12, 2020-March 10, 2020).

\section{Data collection and extraction}

An aggregate number of CT head examinations were collected for the pre and post COVID-19 periods by a neuroradiologist with 5 years of experience (MR). Following this, a crosssectional imaging fellow (MA) reviewed the individual CT reports and collected the indication for the exam and the presence or absence of acute findings that were predefined.

The indications were categorized into the following subheadings: headache, traumatic brain injury (TBI), focal neurological deficit (FND), seizure, ataxia, movement disorder, vertigo, visual complaints, confusion, dizziness/syncope, to rule out non-traumatic intracranial hemorrhage (ICH) and others. The predefined acute imaging findings included acute infarct, acute intracranial hemorrhage, infection, new or enlarging space-occupying lesion(s), new or worsening hydrocephalus, and other acute not specified.

The reviewer (MA) was blinded to the date of the CT examination. In situations where the indication was not clear on the radiology report, that field was left blank and subsequently, our electronic medical record database MEDITECH (Medical Information Technology, Westwood, MA, USA) was searched by the reviewer (MA) at the end of the data extraction process to avoid bias. The number of scans in each category was recorded for the two periods.

\section{Statistical analysis}

A two-sample $t$ test ( $t$ test) was performed as part of the data analysis. A $t$ test was performed to examine the difference in CT head volumes pre-COVID-19 and during COVID-19. $T$ tests were also conducted between each indication category to address differences between the two time points. Lastly, a $t$ test was performed to evaluate for a statistically significant difference in acute findings between the two time periods. Daily averages were calculated for all measures to control for the sample size difference between each time frame. All 
statistical analyses were completed using Microsoft Office Excel (Version 16.40, Microsoft, Redmond, WA, USA), and a $p<0.05$ was considered statistically significant. Assumption checks were performed prior to all analyses.

\section{Ethics approval}

This retrospective cohort study was approved by the research ethics board at our institution. The requirement for obtaining informed consent was waived.

\section{Results}

A total of 772 CT head studies were reviewed. Duplicate order entries $(n=2)$, follow-up imaging during the same visit $(n=$ 14 ), and non-diagnostic studies $(n=5)$ were excluded (Fig. 1). This resulted in a total of 751 scans for review.

There was a statistically significant decrease in the total CT head volume during COVID-19 compared with that during pre-COVID-19 $(p=0.001)$. The volume of CT head studies decreased from 461 scans pre-COVID-19 to 290 scans during COVID-19, making the average number of CT heads per day 16.5 and 10.4, respectively (Table 1, Fig. 2).

The number of CT head scans performed for each indication invariably decreased during COVID-19 compared with that during pre-COVID-19. However, the decrease was statistically significant only for ruling out non-traumatic ICH $(p=$
0.01). There was no scan performed to rule out non-traumatic ICH during COVID-19 compared with 7 scans performed in the pre-COVID-19 period (Table 2).

There was a significant increase in acute intracranial findings on CT heads performed during COVID-19 compared with that during the pre-COVID-19 period $(p=0.001)$. The percentage of CT head studies revealing acute findings increased from $11 \%$ pre-COVID-19 to $23 \%$ during COVID-19 ( $p=0.01 *$, Table 3 ). The percentage of CT head studies revealing nonacute findings decreased from $89 \%$ pre-COVID19 to $78 \%$ during COVID-19, although showed no statistical significance, $p=0.93$ (Table 3, Fig. 3).

Acute findings observed included acute infarct, acute intracranial bleed, infection, new or enlarging space-occupying lesion(s), new or worsening hydrocephalus, posterior reversible encephalopathy syndrome (PRES), calvarial fracture/hematoma, facial fracture/hematoma, facial cellulitis, hyperdense middle cerebral artery (MCA), and a suspected case of Chiari 1 malformation.

\section{Discussion}

In the 4 weeks following the public announcement of our city's first COVID-19 positive case, there was a significant decrease in the overall utilization of emergency CT head services $(37 \%)$ and in the imaging requisitions to rule out nontraumatic ICH (100\% decrease). Interestingly, the yield of CT

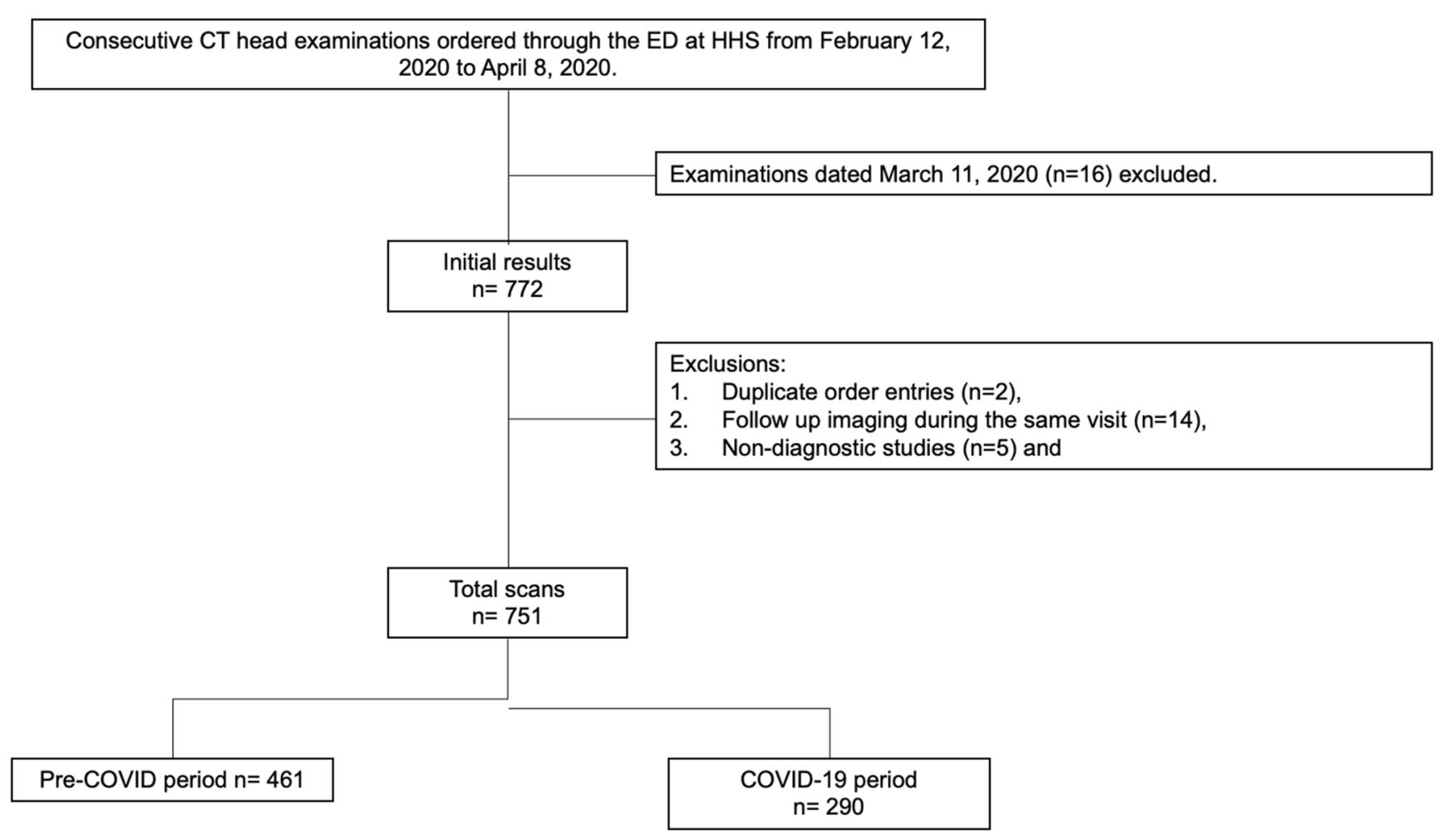

Fig 1 Flow chart of the study population 
Table 1 Study population demographics, total number of CT heads, and average number of CT heads per day in pre-COVID19 and COVID-19 periods (February-April 2020)

\begin{tabular}{llll}
\hline Category & Pre-COVID-19 & COVID-19 & $T$ test results \\
\hline Total number of days & 28 days & 28 days & \\
Mean age (range) & 67 years (18-98) & 68 years (21-99) & \\
Sex distribution (male, female) & 224,237 & 149,141 & \\
Total number of CT scans & 461 & 290 & $t=-9.26, p=1.96 \mathrm{E}-7 *$ \\
Average number of CT scans per day & 16.5 & 10.4 & \\
\hline
\end{tabular}

*Significant test result head examinations for acute findings increased despite the overall reduction in the volume of imaging performed. This is in alignment with a recent Ontario-based study that revealed no change in stroke admissions despite significant reductions in stroke codes in the ED during the early weeks of the COVID-19 pandemic [11]. The collateral effect of the pandemic on healthcare utilization has been a concern for emergency physicians worldwide [4-10, 12, 13]. For example, a recent study from France revealed a substantial decrease in cases of acute stroke (34\%), transitory ischemic attacks (32\%), and seizures (36\%) presenting to the ED and another study from the USA revealed a $39 \%$ decrease in stroke imaging $[12,13]$ However, in our study, the overall reduction in emergency neuroimaging did not result in a decrease in absolute number or proportion of scans with acute findings. This can alleviate, at least to some extent, the fear of "missing out" on severe acute intracranial pathologies requiring urgent medical attention.

Traumatic brain injuries and headaches are not only the most common indications for emergency neuroimaging but are also believed to be common causes for imaging overuse [14-17]. In a study by Sharp et al., about one-third of CT heads performed for head injuries did not lead to any clinical intervention and could be avoided with application of the Canadian CT Head Rule, a validated clinical decisionmaking tool to determine the need for $\mathrm{CT}$ head in adult emergency department patients with minor head injuries [14]. In our study, there was a considerable decrease in imaging referrals for headaches (variance $-47 \%$ ) and head trauma (variance $-40 \%$ ) during COVID-19 compared with that during pre-COVID-19 period whereas referrals for focal neurological deficits varied only slightly (variance $+0.8 \%$ ) (Table 2 ). Such a pattern could result from a number of possible reasons. Firstly, some patients with milder or chronic symptoms either did not seek urgent attention or visited alternative healthcare facilities. This change in behavior can be due to the fear of sharing space with potential COVID-19 patients in larger emergency rooms of a tertiary care center or to avoid overburdening the EDs with non-COVID-19-related ailments. Secondly, there could have been a decline in the incidence of head trauma and headaches due to lifestyle changes during the pandemic. Since people were encouraged to stay home as a measure to limit the spread of infection, the risk of traffic and workplace accidents were expected to decrease. A drop in certain headache precipitating factors like extended work hours and inadequate sleep could also have resulted from such
Fig 2 Trend of emergency department $\mathrm{CT}$ heads by day (February 2020-April 2020)

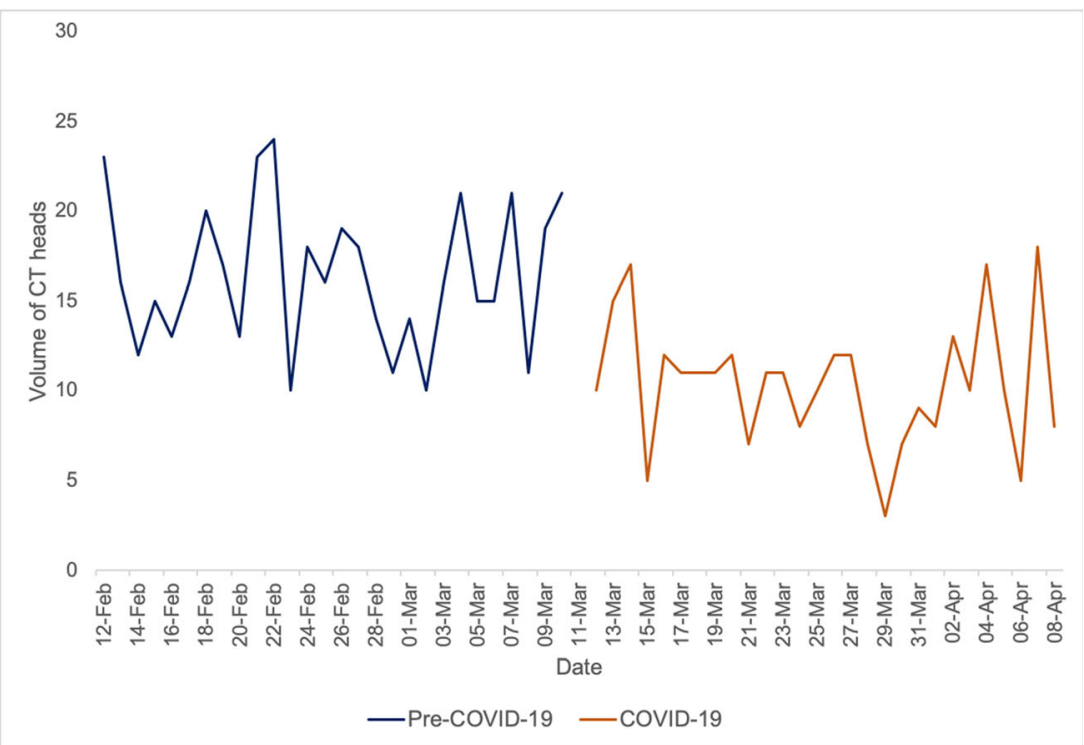


Table 2 Change in CT head referral indications (February-April 2020)

\begin{tabular}{|c|c|c|c|c|c|c|c|}
\hline \multirow[t]{2}{*}{ Category } & \multirow[t]{2}{*}{ Variance test } & \multicolumn{3}{|c|}{ Volumes of referral indication } & \multicolumn{2}{|c|}{ Average number of referral indication } & \multirow{2}{*}{$\begin{array}{l}T \text { test results } \\
\text { (Average) }\end{array}$} \\
\hline & & Pre-COVID-19 & COVID-19 & $\%$ Variance & Pre-COVID-19 & COVID-19 & \\
\hline Headache & Equal & 66 & 35 & -46.9 & 0.15 & 0.12 & $\begin{array}{l}t=1.89 \\
p=0.34\end{array}$ \\
\hline Traumatic brain injury & Equal & 103 & 62 & -39.8 & 0.22 & 0.20 & $\begin{array}{l}t=1.13 \\
p=0.57\end{array}$ \\
\hline Focal neurological deficit & Not equal & 92 & 71 & 0.8 & 0.19 & 0.24 & $\begin{array}{l}t=-2.51 \\
p=0.21\end{array}$ \\
\hline Non traumatic $\mathrm{ICH}^{\mathrm{a}}$ & Not equal & 7 & 0 & -100.0 & 0.01 & 0.0 & $\begin{array}{l}t=4.96 \\
p=0.01 *\end{array}$ \\
\hline Seizure & Not equal & 35 & 19 & -45.7 & 0.08 & 0.07 & $\begin{array}{l}t=0.64 \\
p=0.75\end{array}$ \\
\hline Ataxia & Equal & 6 & 5 & -16.7 & 0.01 & 0.01 & $\begin{array}{l}t=-0.52 \\
p=0.80\end{array}$ \\
\hline Movement disorder & Not equal & 3 & 1 & -66.7 & 0.01 & 0.01 & $\begin{array}{l}t=2.18 \\
p=0.28\end{array}$ \\
\hline Vertigo & Not equal & 19 & 14 & -26.3 & 0.04 & 0.05 & $\begin{array}{l}t=-1.08 \\
p=0.59\end{array}$ \\
\hline Visual complaint & Not equal & 14 & 9 & -35.7 & 0.03 & 0.04 & $\begin{array}{l}t=-0.73 \\
p=0.72\end{array}$ \\
\hline Confusion/altered & Equal & 86 & 53 & -38.4 & 0.19 & 0.19 & $\begin{array}{l}t=0.03 \\
p=0.99\end{array}$ \\
\hline Dizziness/syncope & Not equal & 10 & 8 & -20.0 & 0.02 & 0.03 & $\begin{array}{l}t=-1.09 \\
p=0.59\end{array}$ \\
\hline Others & Not equal & 20 & 13 & -35.0 & 0.04 & 0.04 & $\begin{array}{l}t=-0.30 \\
p=0.88\end{array}$ \\
\hline
\end{tabular}

*Significant test result

${ }^{a}$ ICH intracranial hemorrhage

lifestyle changes. Thirdly, if there may have been a change in the behavior of the ordering clinicians with a more conservative utilization of diagnostic imaging services.

Imaging overuse has been a much talked about topic in the last decade. Choosing Wisely Canada, which started in 2014 as a part of a global movement to limit unnecessary medical tests and treatments, includes recommendations on appropriate imaging in cases of mild head injury or uncomplicated headaches [18]. In our study, there were no scans performed to rule out non-traumatic ICH in the COVID period. Amongst the seven patients scanned for this indication in the pre-COVID-19 period, none of them had an acute intracranial abnormality. This finding could suggest a change in ER physicians' decision-making behavior with respect to ordering

Table 3 Number and proportion of acute and non-acute findings per scan (February-April 2020)

\begin{tabular}{|c|c|c|c|c|c|c|c|}
\hline \multirow[t]{2}{*}{ Category } & \multirow[t]{2}{*}{ Total CT scans } & \multicolumn{2}{|c|}{ Number of CT scans } & \multicolumn{2}{|c|}{$\begin{array}{l}\text { Average proportion of CT scans per } \\
\text { day }\end{array}$} & \multicolumn{2}{|l|}{$T$ test results } \\
\hline & & Acute findings & $\begin{array}{l}\text { Non-acute } \\
\text { findings }\end{array}$ & Acute findings & $\begin{array}{l}\text { Non-acute } \\
\text { findings }\end{array}$ & Acute findings & $\begin{array}{l}\text { Non-acute } \\
\text { findings }\end{array}$ \\
\hline Pre-COVID-19 & 461 & 50 & 411 & 0.11 & 0.99 & $t=-0.46, p=0.01 *$ & $t=0.29, p=0.93$ \\
\hline COVID & 290 & 66 & 224 & 0.23 & 0.80 & & \\
\hline
\end{tabular}

*Significant test result 


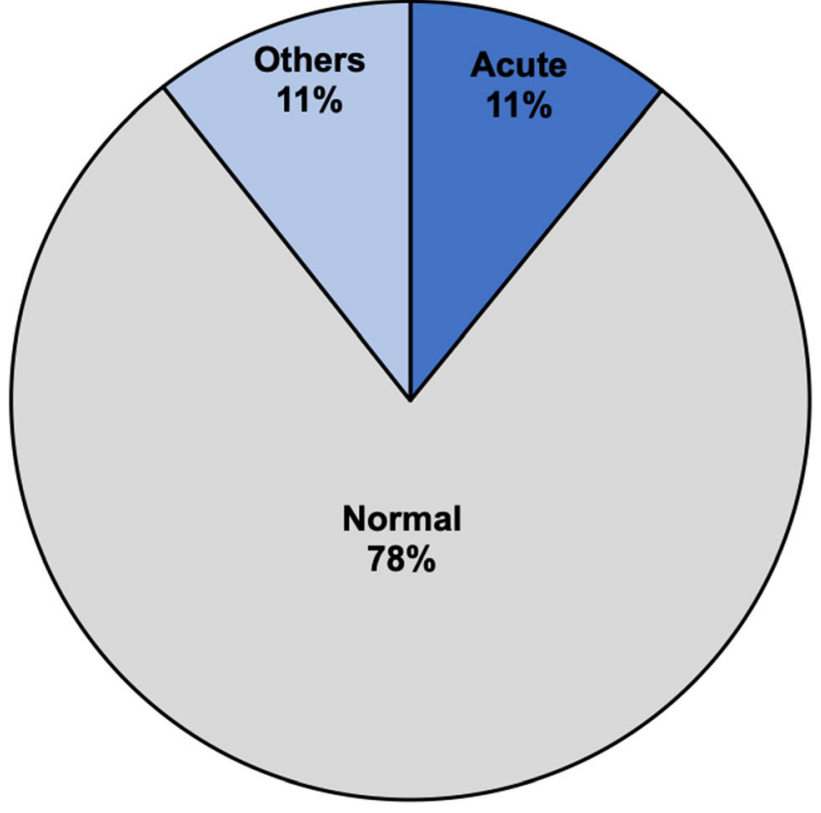

a) Pre-COVID-19

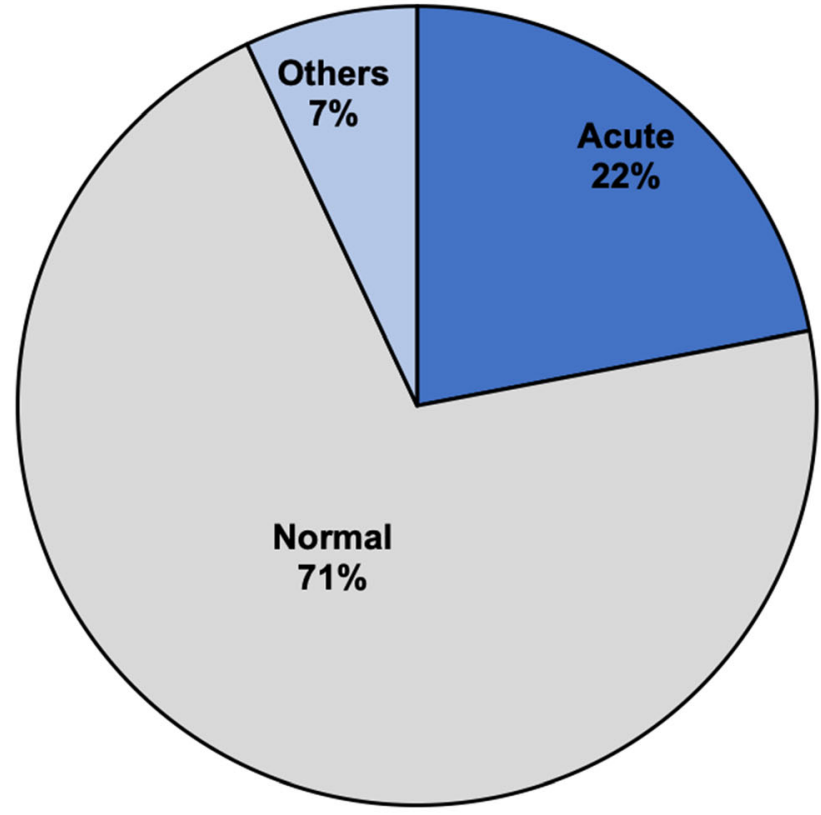

b) COVID-19

Fig 3 Proportion of acute and normal findings noted in emergency department CT heads in pre-COVID-19 period (a) and during COVID-19 pandemic (b)

imaging tests. It could be that the physicians were able to assess every patient in more detail with the overall reduced ED burden or there could have been an attempt to limit the door-to-discharge time for patients without specific or acute neurological symptoms. However, the small size of this subgroup of patients $(n=7)$ limits the strength of this hypothesis.

Nonetheless, the effect on mortality and morbidity due to such behavior change remains unknown. Though the percentage of positive CT head scans requiring specific intervention did not significantly increased during the pandemic, we do not know if these patients presented later with worsened clinical conditions during the pandemic. Besides planning public awareness messages with respect to novel diseases like COVID-19, people's memory regarding the common signs of acute life-threatening diseases like stroke or myocardial infarction also needs to be reinforced during such public health emergencies. Clear communication should be made for uninhibited access for urgent medical help and reassurances need to be given about the safe hospital practices that are in place to limit the spread of infectious diseases.

There are few limitations of this retrospective observational study. Firstly, we used CT head examinations as a surrogate for emergency healthcare access. We did not look at the clinical severity with which patients presented. Secondly, some acute diagnoses like hyperacute stroke or iso-dense masses with no mass effect may be occult on CT. We did not look at MRs performed on an emergency basis as these numbers were small and we did not expect them to yield statistically significant results. Thirdly, our study period was relatively small and included the beginning of the pandemic. This was however this was necessary to appreciate the impact of the pandemic-related fear and response to public communications of staying at home and not burdening healthcare staff before adequate safety measures were in place.

There has been a lot of recent literature in relation to the decline in non-COVID-19-related acute presentations specifically to EDs across many countries. We are yet to find out the actual clinical effect of this trend in terms of mortality and morbidity. Our study shows that patients with acute findings on CT head exams increased during the pandemic while the absolute number of CT exams associated with normal or stable findings declined. It highlights the issue of overprescription in modern imaging along with behavioral change of the public as well as the physicians. While decision support tools are being explored to achieve appropriate imaging, policy-makers need to be more cautious about the public messages that are sent out during infectious disease pandemics like COVID-19.

Author contributions All authors contributed to this manuscript; Drs. Agarwal, Ramonas, M., Alabousi, Ramonas, L., and Van der Pol conceived the study idea and developed the methodology; Drs. Agarwal and Ramonas, M. drafted the protocol and reviewed all data. Dr. Udare contributed to revisions of the protocol. Mr. Mascola designed the cohort identification strategy and conducted the initial search and extraction. Ms. Edmonds performed the primary analysis. All authors contributed to drafting and revisions of the submitted manuscript. 
Data availability Please contact the corresponding author for all study data which can be made available upon request. Data will be shared in accordance with institutional research ethics board protocols.

\section{Compliance with ethical standards}

Conflict of interest The authors declare that they have no conflict of interest.

Ethics approval This retrospective cohort study was approved by the research ethics board at our institution. The requirement for obtaining informed consent was waived.

Code availability Not applicable.

\section{References}

1. World Health Organization (2020) WHO Director-General's opening remarks at the media briefing on COVID-19 - 11 March 2020. https://www.who.int/dg/speeches/detail/who-director-general-sopening-remarks-at-the-media-briefing-on-covid-19\%2D\%2D-11march-2020. Accessed Jun 1, 2020

2. La Grassa J (2020) 14 cancer patients at Hamilton hospital saw doctor who tested positive for COVID-19 | CBC News. CBC.ca. https://www.cbc.ca/news/canada/hamilton/covid19-first-casedoctor-hamilton-health-sciences-1.5493530. Accessed 15 Aug 2020

3. Ontario Provincial Government (2020) COVID-19 Directive \#2 for health care providers (regulated health professionals or persons who operate a group practice of regulated health professionals) issued under section 77.7 of the Health Protection and Promotion Act (HPPA), R.S.O. 1990, c. H.7. Accessed Jun 1, 2020

4. Garcia S, Albaghdadi MS, Meraj PM, Schmidt C, Garberich R, Jaffer FA, Dixon S, Rade JJ, Tannenbaum M, Chambers J (2020) Reduction in ST-segment elevation cardiac catheterization laboratory activations in the United States during COVID-19 pandemic. J Am Coll Cardiol 75:2871-2872

5. Lazzerini M, Barbi E, Apicella A, Marchetti F, Cardinale F, Trobia $\mathrm{G}$ (2020) Delayed access or provision of care in Italy resulting from fear of COVID-19. Lancet Child Adolesc Health 4(5):e10-e11. https://doi.org/10.1016/s2352-4642(20)30108-5

6. Epic Health Research Network (2020) Trends in acute MI and stroke in the ED during COVID-19 - epic health research network. http://ehrn.org/trends-in-acute-mi-and-stroke-in-the-ed-duringcovid-19/. Accessed Jun 1, 2020

7. Thornton J (2020) Covid-19: A\&E visits in England fall by $25 \%$ in week after lockdown. BMJ 369:m1401. https://doi.org/10.1136/ bmj.m1401
8. Santana R, Sousa JS, Soares P, Lopes S, Boto P, Rocha JV (2020) The demand for hospital emergency services: trends during the first month of COVID-19 response. Portuguese J Public Health 38:30 36. https://doi.org/10.1159/000507764

9. Deerberg-Wittram J, Knothe C (2020) Do not stay at home: we are ready for you. Massachusetts Medical Society. https://catalyst. nejm.org/doi/full/10.1056/CAT.20.0146. Accessed Jun 1, 2020

10. Guo H, Zhou Y, Liu X, Tan J (2020) The impact of the COVID-19 epidemic on the utilization of emergency dental services. J Dental Sci. https://doi.org/10.1016/j.jds.2020.02.002

11. Bres Bullrich M, Fridman S, Mandzia JL, Mai LM, Khaw A, Vargas Gonzalez JC, Bagur R, Sposato LA (2020) COVID-19: stroke admissions, emergency department visits, and prevention clinic referrals. Can J Neurol Sci:1-10. https://doi.org/10.1017/ cjn.2020.101

12. Kansagra AP, Goyal MS, Hamilton S, Albers GW (2020) Collateral effect of Covid-19 on stroke evaluation in the United States. N Engl J Med 383(4):400-401. https://doi.org/10.1056/NEJMc2014816

13. Feral-Pierssens A-L, Claret P-G, Chouihed T (2020) Collateral damage of the COVID-19 outbreak: expression of concern. Eur J Emerg Med 27(4):233-234. https://doi.org/10.1097/MEJ. 0000000000000717

14. Sharp AL, Nagaraj G, Rippberger EJ, Shen E, Swap CJ, Silver MA, McCormick T, Vinson DR, Hoffman JR (2017) Computed tomography use for adults with head injury: describing likely avoidable emergency department imaging based on the Canadian CT Head Rule. Acad Emerg Med 24(1):22-30. https://doi.org/10.1111/acem. 13061

15. Klang E, Beytelman A, Greenberg D, Or J, Guranda L, Konen E, Zimlichman E (2017) Overuse of head CT Examinations for the investigation of minor head trauma: analysis of contributing factors. J Am Coll Radiol 14(2):171-176. https://doi.org/10.1016/j.jacr. 2016.08.032

16. Melnick ER, Szlezak CM, Bentley SK, Dziura JD, Kotlyar S, Post LA (2012) CT overuse for mild traumatic brain injury. Jt Comm J Qual Patient Saf 38(11):483-489. https://doi.org/10.1016/s15537250(12)38064-1

17. Gilbert JW, Johnson KM, Larkin GL, Moore CL (2012) Atraumatic headache in US emergency departments: recent trends in CT/MRI utilisation and factors associated with severe intracranial pathology. Emerg Med J 29(7):576-581. https://doi.org/10.1136/emermed2011-200088

18. Canadian Association of Radiologist (2020) Five things physicians and patients should question in radiology. ChooseWiselyCA. https://choosingwiselycanada.org/radiology/. Accessed 15 Aug 2020

Publisher's note Springer Nature remains neutral with regard to jurisdictional claims in published maps and institutional affiliations. 